Jean-Louis Baudouin Professeur à la faculté de droit de l'université de Montréal

\section{Marie-Hélène Parizeau} Chercheur en bioéthique, université de Paris XII

\section{ADRESSES}

M.-H. Parizeau : 63, rue Claude Bernard, 75005 Paris.

J.-L. Baudouin : université de Montréal, faculté de Droit, Case postale 6201, succur-

\title{
Réflexions juridiques et éthiques sur le consentement au traitement médical
}

Le traitement médical est un acte consensuel en France comme aux États-Unis et au Québec. Cette affirmation donne lieu cependant à des " lectures " différentes en France et en Amérique, plutôt paternaliste dans le premier cas et libertaire dans le second.

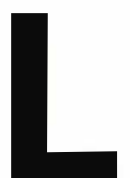

e consentement libre et éclairé du patient est une condition indispensable à l'administration d'un traitement médical comme d'un essai clinique. Dans ce dernier cas cependant, la plupart des systèmes juridiques sont plus exigeants quant à l'intensité de l'obligation de divulgation et requièrent le respect absolu du principe de la proportion des risques par rapport aux bénéfices espérés. Il n'est pas dans notre propos ici d'analyser les problèmes spécifiques du consentement à l'essai clinique (information sur le placebo, le double-insu, etc.), à l'expérimentation humaine (volontaires sains), ou encore à "l'innovation thérapeutique". Nous limiterons donc nos commentaires au consentement au traitement médical proprement dit. Le traitement médical est ici entendu comme l'ensemble des méthodes diagnostiques et thérapeutiques validées, considérées comme les normes courantes dans le but d'améliorer le bien-être d'un patient.

Le traitement médical : un acte consensuel. Aspects juridiques. Aux États-Unis, le modèle recherché de la relation patient-médecin est colui d'une relation égalitaire. L'importance d'un consentement parfaitement informé et éclairé de la part du patient est donc considérable. Pour respecter le caractère individualisé et volontariste de la relation médicale, le médecin doit dévoiler tous les risques probables, tous les traitements alternatifs possibles, toutes les conséquences sérieuses que le traitement est susceptible d'avoir sur la vie et la santé future du patient, bref fournir toute information permettant une expression pleine et entière de la volonté. C'est ce dernier et non le médecin qui choisit le traitement, par respect du principe d'autonomie. Les tribunaux américains se montrent aussi sévères pour le médecin qui a failli à son obligation d'information, que pour celui qui a commis une faute dans l'administration de la thérapeutique. Aux États-Unis, le patient qui doit débourser de sa poche les frais médicaux, se perçoit de plus comme un véritable consommateur de services. Comme dans un contrat de consommation, l'inexactitude dans l'information, ou l'absence de renseignements, sont considérées comme des fautes.

La situation est très différente en 
France. La relation patientmédecin y reste beaucoup plus traditionnelle et revêt davantage une forme de paternalisme (ce mot n'étant pas nécessairement entendu ici dans un sens péjoratif). La relation est aussi plus autoritaire. Cet autoritarisme est toutefois en général accepté par le patient qui, considérant l'homme de science comme un véritable professionnel, admet plus volontiers le caractère inégalitaire des rapports. En France, la relation patient-médecin repose sur un postulat de confiance qui entraîne, par rapport au standard américain, une diminution du contenu et de l'intensité de l'obligation d'information. Le médecin français est donc moins porté (et les tribunaux acceptent bien ce fait) à entrer dans trop de détail et à fournir au malade une information complète. Le Québec, lui, est dans une situation de transition. Pendant longtemps, la relation médicale y a été assez semblable à celle qui existe en France. Toutefois, deux phénomènes sont venus la transformer. Le premier, au début des années 70 a été la prise en charge par l'État du système de soins médicaux et hospitaliers. La relation patient-médecin s'est alors en quelque sorte "fonctionnarisée ". Sous l'influence américaine, le consumérisme a aussi atteint la population québécoise. Ce premier changement a habitué le patient à être plus exigeant et à revendiquer davantage son autonomie décisionnelle, il a forcé le médecin à expliquer les thérapeutiques et à donner une information plus complète. Le second a été la trop rapide évolution de la " médecineart " à la " médecine-technique ". La médecine-technique étant perçue, à tort, comme quasiinfaillible, les patients sont devenus de moins en moins prêts à pardonner au professionnel de la santé l'erreur dans le choix des techniques thérapeutiques ou dans l'interprétation des résultats de tests cliniques.

En France, comme au Québec ou États-Unis, le traitement médical reste cependant considéré par le droit comme un acte consensuel, la plupart du temps contractuel (1), requérant à la fois le consentement du médecin (clause de conscience) et celui du malade. A cet égard, l'attitude du droit et de la médecine à l'endroit du refus de traitement varie considérablement. Aux États-Unis et au Québec (2), le patient est libre en tout temps de refuser tout traitement, même si ce refus entraîne la mort (par exemple le refus de transfusion sanguine du témoin de Jéhovah (3)) à condition de pouvoir donner un consentement éclairé (ce qui exclut l'enfant et la personne handicapée mentale). En France, au contraire, le refus de porter secours à une personne en danger sert souvent à légitimer la position inverse et permet, dans certains cas, l'acharnement thérapeutique, perçu en Amérique comme des voies de fait passibles de sanctions pénales (4). Il existe toutefois une justification encore plus fondamentale à l'exigence du consentement. L'article 19 du Code civil québécois la résume bien dans une formule lapidaire : "La personne humaine est inviolable. Nul ne peut porter atteinte à la personne $d$ 'autrui sans son consentement ou sans y être autorisé par la loi."

Pendant longtemps, les droits jurisprudentiels français, américain et québécois ont suivi des règles identiques. Le traitement médical étant par définition un acte posé pour le bien du patient, les tribunaux, en cas de litige, présumaient aisément que le consentement du patient avait été obtenu et était le fruit d'une décision informée et éclairée. Depuis quelques temps cependant le droit américain et le droit québécois (à un moindre degré) ont évolué dans un autre sens. Pour les raisons déjà mentionnées, les cours de justice scrutent plus volontiers le consentement du patient pour vérifier si tous les éléments d'information nécessaires à la prise de décision ont bien été communiqués et compris. Ainsi, un tribunal a récemment condamné en dommagesintérêts un médecin montréalais qui avait pratiqué sur sa cliente une chirurgie maxillaire importante et n'avait pourtant commis aucune faute opératoire. La patiente, à laquelle la Cour a donné raison, argumentait que le praticien avait fait défaut de lui révéler l'existence d'un autre traitement plus conservateur qui n'aurait pas produit les effets secondaires désagréables qu'elle éprouvait à la suite de l'intervention (5). De même, dans une décision venant d'une autre province canadienne, la Cour suprême du Canada (6) a condamné un médecin qui avait procédé chez son patient à une endartériectomie qui le laissait paralysé et invalide. Le médecin, dans ce cas, avait prévenu son patient que le risque d'attaque était plus grande si l'opération n'était pas tentée. Il avait cependant omis de divulguer que l'opération elle-même comportait des risques sérieux de paralysie. La Cour, suivant la règle américaine, décréta qu'il était du devoir du médecin de prévenir le patient de tous les risques qu'un patient raisonnable, dans la même situation que le demandeur aurait voulu connaître. Ce n'est pas, affirme l'arrêt, parce que la preuve établit le caractère raisonnable de l'opération qu'une personne raisonnable placée dans la situation du patient aurait nécessairement accepté de la subir.

Un tel standard ne manquera pas de faire peur au médecin français qui, comme son confrère québécois jusqu'à récemment, en est resté à une définition plus classique de faute civile: ce qu'un médecin raisonnablement prudent et diligent aurait eu comme conduite dans les circonstances de l'espèce. Il faut toutefois être conscient que les tribunaux français, dans le futur, sans nécessairement suivre le modèle américain, se montreront probablement plus exigeants sur la qualité de la divulgation de l'information médicale. C'est peut-être ce qui explique la tendance actuelle à ne plus se contenter (même si la chose est dans la plupart des cas juridiquement valable) d'un consentement verbal. Le patient, en premier lieu, doit avoir accès à l'information pertinente à sa prise de décision. Le médecin (sauf lorsque la chose 


\section{NOTES}

(1) La décision de la Cour de cassation, Civ. 20 mai 1936 , D. 36.1 .88 , opéra un renversement complet de la jurisprudence. Elle reconnut l'existence d'un contrat entre le médecin et son patient et, par conséquent, l'égalité des parties à ce contrat.

(2) Paragraphe 2, article 42. Loi sur la protection de la santé publique LRQ P-35.

(3) Jurisprudence :

- aux Etats-Unis : Jehovah Witness of the State of Washington King Country Hospital Unit 390 USA 598 (967).

- au Québec : Montréal Children Hospital c Powoll (1975 RP 345). Montréal Children Hospital c Wong (1975 RP 344).

(4) Article 244 et suivants. Code pénal du Canada.

(5) Sunne c Shaw (1981). Rapports de la Cour supérieure 609.

(6) Reibl c Hughes (1980). 2 rapports de la Cour suprême 880 .

(7) Article 42. Loi de la protection de la santé publique du Québec, LRQ chapitre B-35.

(8) Cabanne F., Marin A. "Les droits de l'homme malade devant les nouveaux programmes thérapeutiques. 1. Rapport médical. "Revue des droits de l'homme 1974 ; VII : 515-27.

Jeanson F, Flecheux G, Ghestin P. La responsabilité médicale en psychiatrie. Congrès de psychiatrie et de neurologie de langue française. (78e session. Reims, $23-28$ juin 1980). Rapport de médecine légale. Paris, New York, Barcelone, Milan : Masson, 1980 : 427 p.

(9) Parizeau MH. La pratique des psychiatres en matière de consentement des patients aux essais cliniques. Un consentement libre et éclairé ? Mémoire de DEA, Histoire des Idées et des Mentalités, Université de Paris XII, Val-de- pourrait avoir un effet néfaste direct) doit donc lui divulguer les risques probables du traitement. Ces risques s'apprécient à la fois par rapport à la probabilité de leur actualisation et par rapport à la sévérité de leurs effets. Le médecin doit, en second lieu, adapter la quantité et la qualité de l'information qu'il fournit aux capacités de son patient de les comprendre et de les assimiler. Un vocabulaire accessible, une vulgarisation scientifique est dès lors essentielle, l'obligation d'informer n'étant pas juridiquement satisfaite si l'information n'est pas comprise par le patient. Il est impossible au juriste de fournir au praticien des recettes concrètes et précises de comportement. Le droit ne peut que fixer ici un standard général car tout varie avec les faits particuliers de l'espèce (genre de traitement, gravité des risques, espérance des résultats, chance de réussite, conditions matérielles de l'administration du traitement, état physique et mental du patient, âge, espérance de vie de celui-ci, etc.). Il importe cependant de souligner d'une part que le consentement du patient ne doit pas être considéré comme un simple moment, une simple étape préliminaire à l'initiation de la thérapeutique, mais comme un continuum. Il ne suffit pas au médecin de l'obtenir avant l'administration du traitement. Le consentement (le plus souvent sous forme d'assentiment ou de volonté tacite) doit être présent pendant toute la durée du processus thérapeutique. D'autre part, le consentement à être traité n'est pas nécessairement un consentement aux traitements. Consentir à entrer dans une relation thérapeutique n'est pas donner un mandat en blanc sur le choix et le type de traitement. En fin de compte, le patient est et doit rester le dernier et le seul juge de son intérêt.

L'obtention du consentement implique donc l'obligation pour le médecin de fournir toute information indispensable à la prise de décision, sous une forme compréhensible par le patient, et de s'assurer de la permanence de la volonté pendant toute la thérapeutique.
Pour l'enfant et l'handicapé mental, les solutions françaises, québécoises et américaines se ressemblent. Elles font toutes appel sous des formes différentes au consentement substitué donné par les détenteurs de l'autorité parentale (parents, tuteur) ou par le curateur. Au Québec, un médecin peut fournir les soins et traitements au mineur de quatorze ans et plus sans le consentement du titulaire de l'autorité parentale, sauf si ces soins exigent un hébergement de plus de douze heures ou doivent se prolonger dans le temps (7).

Dimension éthique de la relation médecin-malade.

La notion de consentement à l'acte médical n'est cependant pas d'ordre exclusivement juridique. Pour le droit, elle ne reflète en effet qu'un ensemble complexe de normes sociales, professionnelles et éthiques. Sur le plan éthique, on analysera les pratiques, les motivations, les valeurs sous-jacentes. L'infinie variété des pratiques est liée à la singularité de la relation patient-médecin. En schématisant, on peut opposer deux attitudes extrêmes : parternaliste (plus fréquente en Europe), et libertaire (plus fréquente en Amérique du Nord).

A l'un des extrêmes, la décision appartient au médecin, le rôle du patient est de s'y conformer. Cette attitude s'inscrit dans la relation patient-médecin traditionnelle. Le médecin fait le choix du traitement au nom du patient, pour le plus grand bien de celuici. La décision est communiquée au patient avec autorité (" voici ma prescription "), assortie des informations que le médecin juge utiles. Le patient a la liberté d'accepter ou de refuser la prescription. Il se détermine en fonction de la confiance qu'il accorde au médecin, et hors du cadre de la relation patient-médecin (habituellement les malades n'informent pas leurs médecins de leur "nonobservance"). Le paternalisme éclairé prétend juger du bien du patient mieux qu'il ne peut en juger lui-même. Le consentement du patient est réduit à un simple 
assentiment, basé sur la confiance. Cette attitude est fréquente en France (8). Une enquête menée auprès de psychiatres hospitaliers français a montré qu'environ un sur quatre des psychiatres de l'échantillon ne demandait l'avis de ses patients ni pour les inclure dans un essai clinique, ni d'ailleurs pour une thérapeutique (9). Dénonçant l'infantilisation des patients, des associations de malades et des mouvements consuméristes réclament l'accès direct à l'information : le droit pour chaque personne de consulter son dossier médical (10). Au Québec, ce droit est reconnu par la loi (11). A l'autre extrême, la décision appartient au patient, le rôle du médecin est de communiquer une information complète. Cette attitude est de type " libertaire ": la valeur de liberté individuelle domine toute autre valeur. Le médecin est alors un prestataire de service. Il offre au patient une information maximale sur le diagnostic, le traitement proposé et les traitements alternatifs, leurs taux de réussite respectifs, la liste des bénéfices attendus, les risques et les eff ets secondaires probables ou possibles. Présumé autonome, le patient choisit la stratégie qu'il juge lui-même la meilleure, parmi celles qui sont envisagées. Informé, il décide en connaissance de cause. Juridiquement compétent - supposé rationnel -, il décide en toute liberté, sans aucune contrainte. Le médecin est informé de la décision, prise à l'extérieur de la relation patient-médecin. Le patient peut choisir une solution que le médecin ne juge ni la plus efficace, ni la plus appropriée, voire même qu'il réprouve. Le médecin peut essayer de persuader mais il n'a aucun droit sur le patient (12). La règle est de respecter la liberté d'autrui. Cette attitude libertaire, originaire des États-Unis, a été vigoureusement critiquée par les médecins. Plusieurs études ont mis en évidence la faible mémorisation du patient et sa compréhension parcellaire des explications données par le médecin, que ce dernier utilise ou non un formulaire écrit. D'autres tra- vaux ont souligné l'importance du pouvoir de suggestion du médecin dans sa relation avec le patient (13).

Une troisième attitude existe (14). Elle n'est pas de l'ordre du compromis entre deux extrêmes. Le consentement résulte ici d'une négociation entre deux partenaires, à l'intérieur de la relation patientmédecin. Cette négociation se fonde sur un échange d'information et sur la prise de décision par chacun des intervenants. Le but est de trouver la solution médicale la plus appropriée. Le principe directeur de cette relation est l'égalité des partenaires dans la différence des compétences. La compétence du médecin renvoie à la décision médicale. Son but est le choix d'une thérapeutique. La décision médicale est fondée sur des connaissances approfondies, une expérience pratique " au chevet du malade ", l'évaluation objective et rationnelle de la situation clinique du patient et la capacité de gérer l'incertitude liée au diagnostic et au traitement. Le résultat d'une telle décision est un avis médical sur le traitement jugé le meilleur parmi ceux possibles. La compétence du patient renvoie à l'évaluation de sa situation personnelle en regard de la décision médicale. La maladie, la souffrance physique et la détresse psychologique n'empêchent pas la prise de décision d'un patient (15). Celle-ci résulte de l'évaluation du traitement proposé : est-il acceptable compte tenu de la spécificité individuelle du patient ? Cette évaluation est fondée sur la compréhension des informations médicales fournies, le niveau de confiance accordée au médecin, la perception individuelle de la maladie et de son impact sur son mode de vie, ses valeurs personnelles. La décision du patient est l'acceptation ou le refus du traitement proposé. L'information du patient par le médecin est au départ de cette démarche du consentement. Le médecin explique les avantages, les risques et les effets secondaires, les chances de succès des traitements possibles. Puis il transmet la décision médicale et surtout la justifie, fournissant toutes les explications sur les raisons de son choix. Ce n'est pas une décision d'autorité : elle est présentée come relative. Ce n'est pas la présentation du catalogue des traitements possibles; le médecin fait un choix explicite. L'étape suivante est la décision du patient, suite à son évaluation du traitement proposé. Deux cas de figure se présentent. Dans le premier cas, le plus simple, le patient consent au traitement proposé qui est alors appliqué. Ce consentement n'est toutefois pas un blancseing. Le dialogue entre le médecin et le patient se poursuit constamment tout au long de l'application du traitement. Et si le médecin juge nécessaire de modifier celui-ci, la négociation s'instaure à nouveau : décision médicale, justification du médecin et décision du patient sur l'acceptabilité des nouvelles modalités du traitement. Second cas de figure, le patient refuse le traitement proposé par le médecin. Le patient décide que le traitement ne lui convient pas, par exemple, qu'il ne tient pas compte de ses désirs ou de ses besoins. Ces refus se rencontrent fréquemment dans les cas de maladies chroniques, en gériatrie, en cancérologie (16). Les motivations du refus du patient permettent au médecin de réévaluer, voire de modifier le traitement proposé. Le patient peut alors prendre une nouvelle décision. Le dialogue débouche donc sur une négociation. Celui-ci peut d'ailleurs se conclure par un refus : refus du traitement, refus de traiter. La liberté de refuser est inscrite dans le principe de toute négociation, celle-ci étant fondée sur l'égalité des partenaires.

Cette troisième démarche du consentement n'est ni une décision unilatérale, ni un marchandage, ni une décision conjointe (17). L'information partagée entre le médecin et le patient, en est une condition nécessaire mais non suffisante. C'est une négociation appelant des décisions successives de chacun des partenaires manifestation de leur égalité dans la différence de leurs compétences 


\section{NOTES}

(10) Dossier Médical. Collectif des Usagers de la Santé (CODEMA, CSCV, FENGUS, FNMIP) 23 mai 1986, polycopié : $6 \mathrm{p}$.

(11) Loi sur les services de santé et les services sociaux du Québec. LRQ chapitre S-5.

(12) $T$ Engelhardt va plus loin et affirme qu'essayer de peser sur la décision du patient serait une faute morale. "The Foundations of Bioethics ». Oxford University Press, 1986 : $398 \mathrm{p}$.

(13) Bergler JH, Pennington AC, Metcalfe M, Freis ED. "Informed Consent : How much does the Patient Understand ? Clinical Pharmacology and Therapeutics $1980: 27$ (4) : 435-40. Loftus FF, Fries J. Informed Consent may be hazardous to Health. Science 1979 : 204 (4388), 11.

Ingelfinger FJ. Informed (but Uneducated) Consent. N Engl J Med 1972 ; 287 ; 465-6.

(14) Une telle attitude a été rencontrée chez des diabétologues lors d'une enquête, actuellement en cours, sur le suivi d'un " essai randomisé de la cyclosporine dans le diabète insulino-dépendant *.

(15) Interrogés, des patients dont on venait de diagnostiquer un diabète, ont donné les raisons de leur acceptation ou de leur refus de participer à " l'essai randomisé de la cyclosporine dans le diabète insulino-dépendant ". Les raisons évoquées témoignent d'une compréhension des principaux enjeux de l'essai et d'une évaluation de ses conséquences.

(16) La démarche du consentement, en cancérologie, est possible dans le cadre des unités de soins palliatifs, comme en témoigne l'expérience de centres québécois. "Les soins palliatifs, qu'en pensent les médecins du Québec ? „, Le Médecin du Québec, décembre 1985 : 24-28, 89-90.

(17) Le consentement comme décision conjointe est défendu par certains commentateurs américains tel C. Levine dans une communication orale. * Informed Consent : Can Patients give it ? Can Doctors live with it ? (Oxford University and Hastings Center, Summer Seminar * Medicine, Ethics and Society », 20-27 July

\section{Summary}

The exact content of the notion of consent to treatment is closely related to the social, cultural and legal factors which condition the medical pratice. From a legal point of view, the importance given to the information duty of the doctor changes from a country to another. In the USA, the Courts are as strict for the physician who failed in his information duty as for the one who has committed a fault in administering a therapy. In France, the doctorpatient relationship is based on confidence and this leads to a reduction, in extent and in intensity, of the information duty. The situation in Québec is at the moment transitional. The ethical analysis of consent allows the distinction of two extreme attitudes : one is paternalistic, the other is libertarian. Paternalism fits in with the traditional doctor-patient relationship : the decision is that of the doctor, the patient having to abide by it. In the libertarian situation, the patient is the decision-maker, the doctor's role being to communicate to the patient a complete information. A third attitude is possible : the consent results then from a negociation based on an exchange of information and on decisions taken by each of the participants in order to come to the most appropriate medical solution. The guiding principle of the relationship is the equality of the partners whithin the difference in their competence.

\section{TIRÉS À PART}

M.-H. Parizeau : 63, rue Claude Bernard, 75005 Paris. 